\title{
Adrenocortical carcinoma: a literature review
}

\author{
Ammu Thampi ${ }^{1}$, Ekta Shah ${ }^{2}$, Ghada Elshimy, ${ }^{3,4}$ Ricardo Correa ${ }^{3,4}$ \\ ${ }^{1}$ Loyola Medical Center, South Gate, CA, USA; ${ }^{2}$ Children's National Medical Center, Washington, DC, USA; ${ }^{3}$ University of Arizona College of \\ Medicine-Phoenix, Phoenix, AZ, USA; ${ }^{4}$ Phoenix VAMC, Phoenix, AZ, USA \\ Contributions: (I) Conception and design: R Correa; (II) Administrative support: R Correa; (III) Provision of study materials or patients: None; (IV) \\ Collection and assembly of data: All authors; (V) Data analysis and interpretation: All authors; (VI) Manuscript writing: All authors; (VII) Final \\ approval of manuscript: All authors.
}

Correspondence to: Ghada Elshimy, MD; Ricardo Correa, MD, Es.D. 650 E Indian School Rd., suite 117, Phoenix, AZ 85012, USA.

Email: ghada_elchimi@hotmail.com; riccorrea20@hotmail.com.

\begin{abstract}
Adrenocortical carcinoma (ACC) is reported to be present in $3-10 \%$ of the population with most tumors presenting as benign tumors. Most cases of ACC are a sporadic accumulation of mutations over time. However, studies show a predisposition to various genetic mutations may contribute. Research over the last couple of decades has elucidated causes of ACC to be driven by several molecular changes that include inactivation of tumor suppressor genes and activation of a myriad of different oncogenes, DNA mutations, and epigenetic changes. The widely adopted staging of ACC is by European Network of Study of Adrenal Tumors (ENSAT) due to its correlations with clinical outcomes. At the time of presentation, a detailed history taking with attention to the history of symptoms of hormonal excess and family history of possible hereditary influence is the first step of evaluation. It is followed by a thorough physical examination for evaluation of ACC. Management of ACC poses a unique challenge as it involves oncologic and endocrine issues. Except for one trial, treatment guidelines are based on retrospective studies and non-randomized trials, and therefore the level of evidence is grade II to grade IV. Personalized therapy including identifying the actionable target in each patient is the future of ACC management. The knowledge base of ACC is evolving based on the basic science and clinical trials conducted by worldwide groups such as COMITE of France, ENSAT of Europe, TCGA project and American Australian Asian Adrenal Alliance (A5). Future studies should aim at clear molecular and clinical standardization. Recommended therapeutic strategies should be prospectively recorded.
\end{abstract}

Keywords: Adrenocortical carcinoma (ACC); DNA mutation; metastasis

Submitted Aug 16, 2019. Accepted for publication Nov 01, 2019.

doi: $10.21037 /$ tcr.2019.12.28

View this article at: http://dx.doi.org/10.21037/tcr.2019.12.28

\section{Introduction}

Adrenal tumors are reported to be present in $3 \%$ to $10 \%$ of the population, and most adrenal tumors are small, benign nonfunctioning adenoma. In contrast, adrenocortical carcinomas (ACCs) are rare, lethal malignancies with poor overall survival (1). Most cases of ACC are a sporadic accumulation of mutations over time. However, studies show a predisposition to various genetic mutations may be a contributing factor, especially when the diagnosis is made in children. Moreover, in children, ACC is often present as part of a larger genetic syndrome such as $\mathrm{Li}$ Fraumeni syndrome, Beckwith-Wiedemann syndrome, Neurofibromatosis-1 (NF1), Carney Complex, and Werner syndrome (2).

Other etiological risk factors may include the presence of contraceptive use in women younger than 25 years (due to increased exposure to estrogen) and men who smoke tobacco (3). Additionally, a relative increase in ACC has been noted during pregnancy and may be due to increased estrogen exposure (4). Approximately $10 \%$ to $20 \%$ of adult cases of ACC result in a secondary malignancy $(5,6)$. 


\section{Epidemiology}

The National Institute of Health (NIH) Office of Rare Diseases research reports that fewer than 200,000 ACC cases exist within the United States (US) and that deaths from ACC account for $0.2 \%$ of the deaths annually. The incidence of ACC is 0.72 per million in the USA while 0.5 to 2 per million worldwide (1). Interestingly, South and Southeastern Brazil have a greater than 10- to 15-fold increase in cases of ACCs due to the higher prevalence of tumor protein $\mathrm{p} 53$ (TP53) germline mutation of the tumor suppressor gene allele $\mathrm{R} 175 \mathrm{H}$ and $\mathrm{R} 337 \mathrm{H}$ alleles secondary to a founder effect, with approximately $78 \%$ found in children and $13 \%$ found in adults (7).

When divided further, ACC presents with a bimodal distribution (i.e., in children younger than 5 years and adults aged 40 to 60 years) with an incidence of $1.3 \%$ of all childhood cancers versus $0.02 \%$ to $0.2 \%$ of adult cancers and occurs 1.5 to 2.5 times more in female patients than male patients. Conditions associated with ACC in adults may include Li-Fraumeni syndrome, Familial Adenomatous Polyposis (FAP), Multiple Endocrine Neoplasia type I, and Lynch syndrome $(1,2,5)$.

\section{Genetic predisposition}

Research in recent decades has elucidated the causes of ACC to be driven by several molecular changes that include the inactivation of tumor suppressor genes and the activation of a myriad of different oncogenes, DNA mutations, and epigenetic changes. At the level of the genome, large-scale changes occur in gene expression along with chromosomal aberrations such as chromosomal gains, chromosomal losses, and loss of heterozygosity; DNA methylation; and dysregulation of micro ribonucleic acid (miRNA) leading to overexpression of these sequences. Tumor suppressor genes involved include TP53 and the melanocortin 2 receptor, also known as the adrenal corticotropin hormone receptor (ACTH-R). TP53 is located on $17 \mathrm{p} 13$ and is a key regulator of cell proliferation. TP53 dysregulation plays a key role in many cancers. Amongst patients with ACC, TP53 accounts for both germline and somatic mutations leading to ACC $(5,8-13)$.

Most cases of ACC are sporadic. Around 70\% to $80 \%$ of ACC cases involve TP53 germline mutations, and $20 \%$ to $35 \%$ of ACC cases are due to somatic mutations. Additionally, more than $80 \%$ of somatic mutations related to TP53 mutations are due to a loss of heterozygosity (LOH) at the $17 \mathrm{p} 13$ chromosomal locus. Of the sporadic cases of ACC in children, roughly $50 \%$ to $80 \%$ are due to TP53 germline mutations in both North American and European populations (13). Germline mutations of TP53 also account for $70 \%$ of families with Li-Fraumeni syndrome, a disease characterized by autosomal dominant genetic patterns resulting in increased susceptibility to breast carcinoma, soft tissue sarcoma, osteosarcoma, and ACC $(2,13)$.

$\mathrm{LOH}$ at the $17 \mathrm{p} 13$ locus results in the significant downregulation of two genes found at this locus: Acylcoenzyme A dehydrogenase very long chain and arachidonate-15-lipooxygenase second type within ACC patients compared to those with benign adrenocortical tumors, which has given rise to two hypotheses. The first hypothesis suggests there is a global downregulation of many genes that occur at this locus in ACC, while the second hypothesis suggests there may be other tumor suppressor genes not yet identified. This further suggests that $\mathrm{LOH}$ at $17 \mathrm{p} 13$ occurs as an early event while TP53 somatic mutations are a later event in the development of ACC, which accounts for the differences in frequency of $\mathrm{LOH}$ versus TP53 somatic mutations in the population. Finally, TP53 mutations can affect tetramerization domains, especially the Brazilian subtype R337H mutation, which replaces an arginine to histidine, bringing about $\mathrm{pH}$ dependent effects in different tissues (13).

ACTH-R belongs to a subgroup of 5 receptors of a $G$ protein-coupled receptor superfamily and is encoded on the intronless gene 18p11.26. ACTH-R LOH has been implicated in ACC and nonfunctional adenomas. Furthermore, when quantified, ACTH-R messenger RNA (mRNA) levels are downregulated in ACC as well as nonfunctional adrenal adenomas, whereas it is upregulated in functional adenomas. This suggests a role for ACTH-R in cellular differentiation. Downregulation of ACTH-R, in turn, results in dysregulation of cyclic adenosine monophosphate pathways and dedifferentiation of aggressive tumors $(13,14)$.

By contrast, there is less evidence of somatic mutations in ACC. However, LOH at 11q13 has been shown to occur in $90 \%$ of informative ACCs and $20 \%$ of adrenocortical adenomas (ACA). Furthermore, LOH across the 11q domain has been documented in ACC, which suggests the involvement of other tumor suppressor genes. To summarize, the documented various oncogenic alterations were considered drivers of tumorigenesis in ACC as they play a critical role. Most notable is the overexpression of insulin growth factor II (IGF-II), which has occurred in 
$60 \%$ to $90 \%$ of ACC cases with a 100 -fold increase in IGFII $(1,2,5,9,13)$.

IGF-II is a fetal growth factor that plays a role in the development of the adrenal cortex. The $11 \mathrm{p} 15$ chromosome locus is arranged into a telomeric domain-the IGF-II cluster, H19-a noncoding region of $2.3 \mathrm{~kb}$, and the centromeric domain-CDKN1C1. The H19 gene is not translated but may play a role in the modulation of IGF-II expression, and CDKN1C gives rise to a cyclin-dependent kinase that regulates the cell cycle at the G1/S phase. IGFII and $\mathrm{H} 19$ are regulated by an imprinting center known as IC1, which is methylated on the paternal allele and unmethylated on the maternal allele. Furthermore, IGF-II is only expressed on the paternal allele, while H19 is only expressed on the maternal allele. Therefore, IGF-II will normally be regulated by methylation of the paternal allele and expression of H19. CDKN1C, however, is regulated by another imprinting center known as IC2. Additional oncogenic alterations implicated in ACC include the constitutive activation of the $\mathrm{Wnt} / \beta$-Catenin pathway due to genetic alteration of $C T N N B 1$ gene and Steroidogenic Factor 1 (SF1) gene overexpression. The role of CTNNB1 gene mutations resulting in $\mathrm{Wnt} / \beta$-Catenin pathway activation was first discovered in FAP patients presenting with ACC and plays a role in embryonic development and development of the adrenal cortex $(13,14)$.

Since the initial studies, transcriptome studies have found Wnt/ $\beta$-Catenin target genes-BIRC5, ENC1, PTTG1, and TWIST1-to be overexpressed in ACC cases. Additionally, CLDN1, AXIN2, and LGR5-positive transcriptional target genes-are overexpressed in ACC patients, giving further evidence to the role of Wnt/ $\beta$-Catenin in tumorigenesis of ACC patients. Current research further suggests a role for $\mathrm{ZNRF} 3$ as a driver for ACC tumorigenesis in altering the $\mathrm{Wnt} / \beta$-Catenin pathway. ZNRF3 encodes for a cell-surface transmembrane E3 ubiquitin ligase that serves as a negative feedback of the Wnt/ $\beta$-Catenin pathway by preventing complex formation between a Wnt receptor known as Frizzled and its coreceptor low-density lipoprotein LRP5/6, which signals for $\beta$-Catenin to be phosphorylated by GSK3, which, once again, leads to degradation of $\beta$-Catenin by the E3 ubiquitin pathway. However, in ACC patients, an inactivating mutation of ZNRF3 has been reported with a $19 \%$ to $21 \%$ prevalence, leading to the activation of $\beta$-Catenin and its subsequent translocation to the nucleus (14). SF1 is a transcription factor that is normally expressed in the hypothalamus, pituitary, and steroidogenic hormone- producing glands such as the ovaries, testes, and the adrenal cortex, playing a crucial role in the development of these glands. Specifically, SF1 has a role in both steroidogenesis as well as fetal development of the adrenal cortex independent of the processes of steroidogenesis (15). Its etiological role in the molecular pathogenesis of ACC is explained by examining its cellular context in benign adrenocortical neoplasms such as ACA versus its cellular context in ACC. In ACAs, the cellular context of SF1 expression is to follow a differentiated cellular phenotype following a pattern that is dependent on steroidogenesis, whereby most steroidogenic enzymes possess SF1 response elements in its promoter region. On the other hand, in ACC, it follows a phenotypical pattern that is like fetal development independent of steroidogenesis, whereby the cellular context of the tumors is less differentiated and does not correlate with hormonal activity. Furthermore, most childhood cases and many adult cases of ACC are associated with overexpression of the SF1 and that there is a decreased survival in the presence of SF1 overexpression. Patients with ACC with high presence of SF1 had a 14-month survival while those with low SF1 presented with 49.8-month survival, further supporting SF1 as a sign of poor prognosis (15).

Evidence has been found for the molecular changes between ACC in relation to Lynch Syndrome, Carney Complex, and Neurofibromatosis Type I. Global gene expression studies have identified a myriad of genes as drivers of pathogenesis in ACC. This includes ZNRF3 and PRKAR1A. Additionally, this includes RPL22, TERF2, CCNE1, NF1, and TERT9. The TERT gene encodes for telomerase and is amplified in ACC cases with a $15 \%$ prevalence, and mutations in DAXX and ATRX genes (playing a role in the lengthening of telomeres) have been found to have a $10 \%$ prevalence in ACC. CDK4 is another amplified gene. Moreover, there have been homozygous deletions of CDKN2A, RB1, ZNRF3, 3q13.1, and 4qe4.3 loci. Global gene expression uses levels of BUB1B and PINK1 in assessing survival of patients with malignant ACC tumors regardless of tumor stage, and DLG7 and PINK1 levels are markers of disease-free survival regardless of tumor grade as will be discussed further in the prognosis section $(14,16)$.

Chromosomal aberrations leading to chromosomal gains or chromosomal losses have also been reported in the pathogenesis of ACC. Initial studies showed chromosomal gains occur at the following loci: 4q, 4p16, 5p15, 5q12-13, $5 q 32$-qter, $9 \mathrm{q} 34,12 \mathrm{q} 13$, and $19 \mathrm{p}$, while chromosomal losses occur at 1p, 21, 11q, 17p, 22p, and 22q1. Further studies 
have since confirmed chromosomal gains at chromosomes 5, 7, 12, 16, 19, and 201,7 and chromosomal losses at 1, 2, 13, 17, and 221,7. Lastly, microsatellite studies indicate $\mathrm{LOH}$ occurs at the following loci: $17 \mathrm{p} 13,11 \mathrm{q} 15$, and 2 p16 at a rate of $85 \%, 90 \%$, and $92 \%$ of ACC cases, respectively. DNA methylation has also been studied at a genome-wide level in ACC; there was significantly more hypermethylation at $\mathrm{CpG}$ sites ( $\mathrm{CpG}$ islands) in $\mathrm{ACC}$ patients versus those with normal adrenocortical tissue samples. The sites of hypermethylated $\mathrm{CpG}$ islands were in regions that led to decreased expression of genes involved in cell cycle regulation and apoptosis regulation. This includes CDKN2A, GATA4, DLEC1, HDAC10, PYCARD, and SCGB3A1/HIN17. The hypermethylation of promoter regions that play a role in downregulation of expression of various genes and hypermethylation of H19 and other tumor suppressor genes (as described above) has also been found in two separate studies $(5,14,16,17)$.

Dysregulation of miRNA has been reported in patients with ACC, leading to the inactivation of tumor suppressor genes and the activation of oncogenes. Further studies have found miR-483-5p located in an intron of IGF-II to play a significant role in ACC pathogenesis. Dysregulation of IGF-II disturbs the expression of miR-483, leading to its upregulation - an event that has occurred in a significant number of pediatric ACC cases. Additionally, the upregulation of miR-1202 and miR-1275 has also been implicated. Similarly, studies have shown downregulation of miR-214, miR-375, miR-511, miR-195, miR-99a, and miR-100, the latter two of which play a role in pediatric $\operatorname{ACC}(5,14,16,17)$.

\section{Clinical presentation}

ACC can present classically in three different forms. Approximately $40 \%$ to $60 \%$ of the patients present with symptoms of hormonal excess, one-third present with nonspecific symptoms, and $20 \%$ to $30 \%$ are diagnosed with imaging studies performed for unrelated medical issues $(5,6,18,19)$. Hormonal excess could be due to hypercortisolism or hyperandrogenism. Hypercortisolism can present as a plethora, diabetes mellitus, osteoporosis, and muscle atrophy. Glucocorticoid-mediated mineralocorticoid receptor activation due to saturation of the HSD11B2 system can present with hypokalemia and hypertension. Malignant adrenal tumors usually present with the clinical presentation of hypercortisolism, rapidly progressing muscle weakness, and pronounced muscle weakness.
Hyperandrogenism can present with male pattern baldness, virilization, hirsutism, and menstrual abnormalities. About $1 \%$ to $3 \%$ of malignant adrenal tumors have excess estrogen production that present as gynecomastia and testicular atrophy. Isolated hyperandrogenism in male patients is often underdiagnosed. Autonomous aldosterone secretion that presents as hypertension and hyperkalemia are rare in ACC. Nonspecific symptoms include local tumor growth, abdominal or flank pain, early satiety, and abdominal fullness $(5,6,20)$. The average size of the tumor at diagnosis is approximately 10 to $13 \mathrm{~cm}(5,21)$. Only a minority of them present $<6 \mathrm{~cm}$, and only $3 \%$ of the cases present as tumors $<4 \mathrm{~cm}(5,21)$. Typical symptoms of cancer such as cachexia and night sweats are rare. Although paraneoplastic symptoms are also rare, tumor-related, IGF-2 mediated hypoglycemia (i.e., Anderson's syndrome) is a common symptom. Tumor chemokines can also cause hyperreninemic hyperaldosteronism, erythropoietinassociated polycythemia, and leukocytosis. ACC can also cause deep venous thrombosis and pulmonary embolism. Up to $70 \%$ of tumors present with biochemically apparent adrenocortical hormone production. Imaging studies have improved the rate of early diagnosis of ACC that present with other medical conditions $(5,6)$.

\section{Staging}

The staging system for ACC, introduced by the World Health Organization in 2004, was based on the modifications on the traditional McFarlane by Sullivan (Table 1). However, it is not widely used due to severe shortcomings. The widely adopted staging of ACC is by the European Network for the Study of Adrenal Tumors (ENSAT) due to its correlations with clinical outcomes (Table 2) $(5,18,22)$.

\section{Differential diagnosis}

The main differential for hypercortisolism includes ACTH independent cortisol-producing adenomas and ACC. The mineralocorticoid excess can be due to bilateral adrenal hyperplasia or adrenocortical autoantibodies. Hyperandrogenemia can be seen in the polycystic ovarian disease, ovarian hyperthecosis, and congenital adrenal hyperplasia. The NIH has established guidelines for evaluating incidentally discovered adrenal mass. For adrenal lesions $>4 \mathrm{~cm}$, the main differential diagnosis includes ACA, myelolipoma, adrenal metastasis of another tumor, 
Table 1 WHO TNM staging of ACC $(16,17)$

\begin{tabular}{lll}
\hline Stage & Description & Relation to adrenal gland \\
\hline Stage 1 & Tumor size is $\leq 5 \mathrm{~cm}$ & Confined within adrenal gland \\
Stage 2 & Tumor size $>5 \mathrm{~cm}$ & Confined within adrenal gland \\
Stage 3 & & Tumor spread to the surrounding tissue including para adipose tissues and adjacent organs \\
Stage 4 & Distant metastasis \\
\hline
\end{tabular}

ACC, adrenocortical carcinoma; TNM, primary tumor, lymph node, distant metastasis; WHO, World Health Organization.

Table 2 ENSAT TNM staging of ACC $(16,17)$

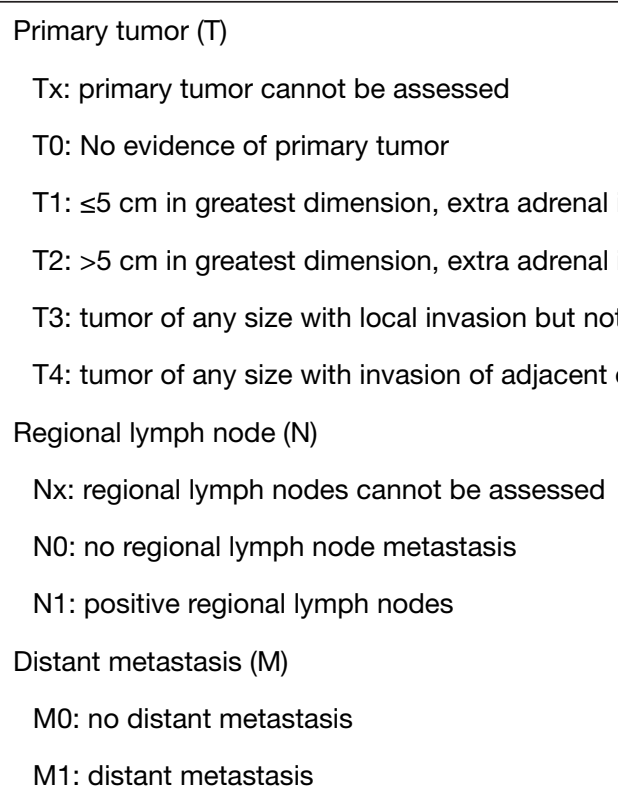

ACC, adrenocortical carcinoma; ENSAT, European Network for the Study of Adrenal Tumors; WHO, World Health Organization.

pheochromocytoma, adrenal cyst, ganglioneuroma, sarcoma, and lymphoma. Adrenal cysts could be a cystic ACC, benign cysts such as bronchogenic or retroperitoneal cyst, and cystic pheochromocytoma. Adrenal pheochromocytoma can be differentiated biochemically. Except for adrenal lymphoma, large adrenal tumors are further evaluated by surgical resection $(23,24)$.

\section{Management}

\section{Evaluation}

At the time of presentation, a detailed history taking with attention to the history of symptoms of hormonal excess such as cushingoid features, hirsutism, and hypertension and family history of possible hereditary influence is the first step of evaluation. It is followed by a thorough physical examination for the evaluation of ACC (5).

\section{Biochemistry}

Biochemical testing helps to rule out the diagnosis of hormonal excess, helps to decide if further invasive procedures are needed to rule out the adrenocortical origin of the tumor, differentiate between androgen or estrogen production, to adopt steroid hormones as tumor markers for follow-up and surveillance, and to decide on postsurgical hydrocortisone replacement therapy. Blood and urine tests help to identify functional versus nonfunctional tumors. Measurement of steroid hormones is the hallmark for the biochemical evaluation of the ACC. Hypercortisolism 
is measured by a $1-\mathrm{mg}$ dexamethasone suppression test, midnight salivary cortisol, or 24-hour urine cortisol. Aldosterone production is measured via plasma renin activity and serum aldolase levels. Hyper androgen production is measured by dehydroepiandrosterone sulfate and total or available testosterone levels. Pheochromocytoma is measured via 24-hour urine or plasma metanephrine and normetanephrine. Measurement of other enzymes is debatable. It is inconclusive whether urinary gas chromatography-mass spectroscopy steroid profile would provide superior diagnostic accuracy. Earlier urinary androgen and androgen precursors such as 11-deoxycortisol metabolites, and dehydroepiandrosterone were used as tumor markers (25). Recently, Arlt et al. reported that the levels of urinary steroid metabolites and precursors were significantly different in benign adrenal tumors versus ACC, and 11-deoxycortisol metabolite tetrahydro 11-deoxycortisol is the most useful tumor marker for surveillance and follow-up of ACC (26).

\section{Imaging}

Contrast-enhanced computed tomography (CT) or magnetic resonant imaging (MRI) are the standard imaging modality for diagnosis, staging, and follow-up. Functional imaging using positron emission tomography (PET), fluorodeoxyglucose (FDG), and mitotane might be used. Tumors sized 3 to $10 \mathrm{~cm}$ can be diagnostically challenging with imaging techniques; some present as incidentaloma. A heterogeneous adrenal mass $>4 \mathrm{~cm}$ with any imaging characteristics of malignancy should undergo complete imaging and surgical evaluation. Ultrasound imaging is valuable to detect liver metastasis and follow-up. All suspected patients are required to undergo a chest $\mathrm{CT}$ to rule out pulmonary metastasis (12).

\section{CT and MRI}

CT with delayed contrast media washout with a $50 \%$ washout cut-off and an absolute value of $>35$ Hounsfield unit (HU) after 15 minutes have superior diagnostic accuracy than other means (12). If $\mathrm{HU}$ is $<10$, this indicates a benign adenoma according to studies published in the last 15 years. ACC presents as a large heterogeneously enhancing mass of soft-tissue attenuation. Two to $10 \%$ of the cases are bilateral $(6,17)$. Specific findings in CT include large mass size, high density (i.e., $>10 \mathrm{HU}$ ), intramural necrosis, and irregular shape and margins (13). Metastatic tissues can be found in the liver, lungs, lymph nodes, renal vein, and inferior vena cava. Nine to $19 \%$ of cases present with inferior vena cava invasions. ACC appears to be hypointense to isointense relative to liver parenchyma on $\mathrm{T} 1$ weighted images and hyperintense relative to T2 weighted images on MRI. Irregular peripheral heterogeneous enhancement with central non-enhancing area secondary to hemorrhage or necrosis is usually found in contrast-enhancing imaging. CT imaging also captures calcifications in almost $30 \%$ of cases as high attenuating foci (22). Intracellular lipid and macroscopic fat are also found in some conditions. While contrast-enhanced CT is an ideal imaging modality for disease staging and metastatic spread, MRI with its multiplanar capability is better equipped for identifying direct invasion of adjacent organs (27).

\section{$\left[{ }^{18}\right.$ F]FDG PET/CT imaging}

Groussin et al. reported a study of 77 patients, which showed that $\left[{ }^{18} \mathrm{~F}\right] \mathrm{FDG}$ PET/CT imaging has a sensitivity of $100 \%$ and a specificity of $88 \%$, differentiating benign and malignant adrenal tumors with a cut-off value of 1.45 for adrenal-to-liver maximum standardized uptake value. It is useful in staging ACC and evaluating local recurrence. Still, it is a complementary imaging modality due to its limitations such as the inability to distinguish between adrenal versus nonadrenal masses and detecting small adrenal lesions (28).

\section{Newer imaging techniques under investigation}

Iodometomidate is being considered as a highly specific tracer for adrenocortical tissue imaging (29). Proton magnetic resonance spectrometry is another modality that is being studied for differentiating ACC from other lesions with higher accuracy than conventional means (22).

\section{Pathology}

The main distinctive feature of ACC is an invasion of the tumor into the adrenal soft tissues, lymphatic channel, and the blood vessels, which can extend up to the inferior vena cava and the right side of the heart. Other features include changes in growth pattern, the presence of tumor necrosis, increased mitotic rate, and the presence of atypical mitotic figures. Reticulin staining is used to highlight the disruption of the reticulin network. Proliferation immunomarkers such as Ki67 are also used for the diagnosis of ACC. 
Immunostains that provide adrenocortical differentiation include alpha inhibin, calretinin, synaptophysin, melanin A, and steroidogenic factor (30).

\section{Molecular pathology}

Using the transcriptome analysis to detect genes expressed can discriminate ACC from ACA. It also helps differentiate carcinoma into various prognostic groups. For stage 1 to stage III ACC, molecular classification can better discriminate poorer outcomes of ACC (30). For histopathological diagnosis, SF1 has emerged as the most valid marker $(15,17)$. A proliferation index, such as Ki67 immunomarker or mitotic count, can help to define the diagnosis and prognosis of ACC, and recently, studies showed that Ki67 is a powerful prognostic marker for local and advanced ACC to guide treatment decisions $(31,32)$.

\section{Clonality}

Genetic assessment with flow cytometry has proven that aneuploidy was observed in ACC tumors (33). The assessment of aneuploidy with histopathologic criteria of 9 adrenal tumors revealed 7 of them had a high Weiss score of $>3$, indicative of malignancy (34).

\section{Chromosomal aberrations}

Comparative genomic hybridization studies have proven that genetic diversity due to loss and gain of chromosomes such as genomic aberrations in chromosome 5, 12, and 17 are predicted to harbor genes and initiate neoplastic transformation $(35,36)$.

\section{Epigenetic variations}

Hypermethylation of promoters and silencing of genes H19, PLAGL1, G0S2, and NDRG2 is correlated with poor survival in ACC (36).

\section{Gene expression profiling}

Global gene expression studies have identified that expression levels of BUB1B and PINK1 alone help in identifying different subgroups of ACC with different survival rates, and expression levels of DLG7 and PINK1 help in identifying subgroups of ACC with distinct diseasefree survival irrespective of their tumor staging $(37,38)$.

\section{MicroRNAs}

miRNAs are small, noncoding 18- to 25-nucleotide RNAs vital in posttranscriptional regulation of gene expression and various cellular processes such as proliferation, apoptosis, and differentiation. Studies have reported that upregulated miRNA in ACC includes miR-184, miR-210, and miR-503 and down-regulated miRNA includes miR-214, miR-375, and miR-511. To differentiate between benign and malignant adrenal lesions, the determination of the level of miR-184, miR-503, and miR-511 can be used. They have $100 \%$ sensitivity and up to $97 \%$ specificity (39). In pediatric studies, miR-483, located in the intron of the IGF2 was found to be upregulated while miR-99a and miR-100 that target the 3'-untranslated regions of IGF1R, RPTOR, and FRAP1 (mTOR) was found to be downregulated (40).

\section{Prognosis of ACC}

The prognosis of ACC was previously believed to be quite poor. Some studies estimate an average overall survival in patients with ACC is 14.5 months with a 5 -year mortality rate of about $75 \%$ to $90 \%$, and median survival for metastatic disease (stage IV) at the time of diagnosis is $<1$ year. Another study reports a $35 \%$ to $58 \% 5$-year survival rate for ACC, while yet another study reports $37 \%$ to $47 \% 5$-year survival rate. Furthermore, it is worth noting that only about $30 \%$ of ACC cases are curable at early stages, which further implied the overall poor prognosis of $\operatorname{ACC}(1,11,12)$.

Metastases of ACC has been detected in the liver in $48 \%$ to $85 \%$ of cases, in the lungs in $30 \%$ to $60 \%$ of cases, lymph nodes in $7 \%$ to $20 \%$ of cases, and bones in $7 \%$ to $13 \%$ of cases (12). Of note, however, was the poor sample size of ACC patients due to the relatively smaller prevalence of patients, which made ascertaining prognosis more difficult in the past. More recently, different studies have shown that ACC clinical presentation, survival, and prognosis is quite heterogeneous, ranging from shortterm to long-term survival. The Union for International Cancer Control and the ENSAT each developed different staging systems. This work led to the understanding that three main criteria determine disease-free survival in stage I-III ACC cases and overall survival in stage IV ACC, as follows: (I) staging; (II) resection status; (III) grading of the tumor based on Ki67 proliferation index and mitotic count $(14,41,42)$.

Based on the ENSAT staging system, there was a $66 \%$ to 
$82 \% 5$-year survival rate in patients with stage I ACC, $58 \%$ to $64 \% 5$-year survival rate in patients with stage II ACC, $24 \%$ to $50 \% 5$-year survival rate in patients with stage III ACC, and $0 \%$ to $17 \% 5$-year survival rate in patients with stage IV ACC $(14,41,43)$. Similarly, resection status was shown to play a key role in the disease-free survival of localized ACC cases. Patients who had completely resected (R0) localized tumors were less likely to have a recurrence. By comparison, those with incomplete microscopic resection (R1), incomplete macroscopic resection (R2), or unknown resection $(\mathrm{Rx})$ were associated with overall survival of $20 \%$ and $15 \%$, respectively. Finally, tumor grade plays a key role in understanding the prognosis of ACC $(14,41)$.

In 2018, Tella et al. obtained data from the National Cancer Database of 3,185 patients with ACC to assess the survival outcomes and predictors of survival. They found an improved survival with surgical resection of the primary tumor, irrespective of disease stage; however, postsurgical chemotherapy or radiation was of benefit only in stage IV disease. Also, lymphadenectomy was associated with improved overall survival in stage IV disease and was not associated with survival benefit in stage I to stage III disease (44).

European studies of ACC patients have shown that the proliferation index, Ki67, has been the single most important factor in determining the recurrence of ACC in patients following complete resection of a localized ACC tumor (R0) for stage I-III ACC patients. Further research suggests that stage I-III, younger age, and low Ki67 are positive predictors of recurrence-free survival (42). Similarly, another large European study showed that tumor grading as measured by Ki67 and the Weiss score were considered important prognostic markers of overall survival in stage IV ACC patients, which further confirmed data shown by mitotic count in French studies (41). By comparison, in adults, cortisol production, higher tumor stage (i.e., advanced stage III/stage IV), and more disordered tumor grade have been identified as negative prognostic factors in ACC with regards to overall survival and recurrence of the disease (14). Moreover, older age ( $>45$ years), distant metastasis, and incomplete resection have been shown to be associated with poor survival (11).

ENSAT staging of ACC led to better predictive value in the prognosis of ACC patients. However, it still presented with some pitfalls such as not accounting for severe invasion into the inferior vena cava or $\mathrm{N}$ status (i.e., Nodal invasion), both of which were found to behave in a pattern more like stage IV ACC (42). Therefore, a modified ENSAT staging system was developed to evaluate advanced stage III to stage IV ACC cases. In this modified ENSAT staging system, the presence of positive $\mathrm{N}$ status moved from stage III to stage IV. The modified ENSAT stating system also accounted for the number of tumor organs and GRAS factors such as tumor grade (Weiss score $<6$ or $>6$ or Ki67 $<20 \%$ or $>20 \%$ ), resection status of the primary tumor (R0, R1, R2, Rx), age ( $<50$ or $>50$ years old), and presence or absence of tumorrelated or hormone-related symptoms at time of diagnosis (42). These criteria have been shown to play key roles in the determination of overall survival as well as in recurrence of stage I-III localized ACC cases (42).

Recent studies have focused on further understanding the molecular pathophysiology of ACC to look for molecular markers that may further provide evidence as prognostic factors. Some candidates in this direction include molecular drivers of ACC such as TP53, ZNRF3, and $\beta$-catenin, as well as looking at hypermethylation status and microRNA profiles such as miR-483-5p. TP53, SF1, and CpG islands with extensive hypermethylation have all been shown to be affiliated with poor or impaired prognosis. Clustering of DNA methylation profiles defined two groups: $\mathrm{CpG}$ island methylator phenotype (CIMP) and non-CIMP. The CIMP group was further classified into high CIMP and low CIMP. Hypermethylation proved to be an important prognostic factor for both disease-free survival and overall survival of ACC patients. This was further shown in the multivariate analysis that included ENSAT staging and Ki67 proliferation index. Furthermore, high CIMP phenotypes were also shown to be found in $\mathrm{C} 1 \mathrm{a} / \mathrm{x}$ and $\mathrm{Ca} 1 /$ p53 tumors (aggressive tumor subgroups) versus the nonCIMP phenotype which was observed in $\mathrm{C} 1 \mathrm{a} / \mathrm{b}$-catenin and C1b tumors (14).

Similarly, the miRNA sequences miR-195 and miR483$5 \mathrm{p}$ have been shown in serum to serve as biomarkers of aggressive ACC, associated with poor prognosis $(14,17)$. By comparison, IGF-II, H19, and CDKN1C have shown no clear relationship to prognosis due to the near-uniform overexpression of IGF-II in ACC cases, accounting for $90 \%$ of ACC cases $(14,17)$. miRNA profiles give rise to 3 stable tumor clusters associated with different hierarchical clustering patterns of miRNA expression profiles, and each are associated with a different prognosis. Mil tumors had the largest difference of miR expression in normal adrenal tissue along with Mi2 cluster tumors and $\mathrm{C} 1 \mathrm{~b}$ tumors (i.e., less aggressive tumors with better prognosis and outcomes). Mi3 clustering belonged to C1a tumors, which were more 
aggressive proliferating tumors with poor prognosis (14).

More recently, transcriptome data have ascertained a relationship between the expression of DLG7 and PINK1 with disease-free survival, overcoming the uncertainties of intermediary Weiss score classified tumors. Likewise, BUB1B and PINK1 were shown to be the best predictors of overall survival in more aggressive/malignant tumors. Additionally, there has been a correlation between TOP2A, $\mathrm{Ki67}$, EXH2, and cyclin B1 staining with overall survival. The presence of a precise genetic pattern (i.e., large chromosomal gains at 5, 7, 12, and 19 and chromosomal losses at 1, 2, 13, 17, and 22) are associated with better prognosis. Three different subgroups of C1a tumors consisted of TP53 mutations (C1a/p53), $\beta$-catenin cytoplasmic, and nuclear accumulations (C1a/ $\beta$-Catenin), and $\mathrm{C} 1 \mathrm{a}$ tumors with mutations that were mutations other than $\mathrm{p} 53$ or $\beta$-Catenin mutations $(\mathrm{C} 1 \mathrm{a} / \mathrm{x})$. Although all three subgroups followed different tumorigenesis processes, they were equally associated with poor prognoses $(14,41)$.

\section{Treatment}

The management of ACC poses a unique challenge as it involves oncologic and endocrine issues. Except for 1 trial, treatment guidelines are based on retrospective studies and nonrandomized trials, and, therefore, the level of evidence is grade II to grade IV (45).

\section{Surgery}

The mainstay of treatment for ACC is surgery. All patients with stage I or II and most with stage III should be amenable to radical resection. Only half of the diagnosed ACC cases have potentially resectable tumors. A radical resection induces a temporary cortisol deficiency in patients with cortisol-secreting ACC who require glucocorticoid replacement postoperatively. Debulking surgery helps with hormonal control before initiating systemic therapy. During surgery, standard oncologic resection practices (e.g., en bloc adrenal gland resection, margin-free resection, no tumor spillage, and conversion to laparotomy in case of difficult dissection) must be duly followed. In the case of recurrent cases, surgery is recommended for patients with favorable prognosis, including a disease-free interval of longer than 6 to 12 months, and an achievable R0 resection. Surgery for palliative and metastatic cases is considered according to prognostic factors. Expected postoperative mortality for patients with ACC is $<5 \%$. Re-surgery is recommended for R2 resection. Locoregional lymph node resection may improve diagnostic accuracy and therapeutic outcome. A multivariate analysis found a significant reduction in the risk of tumor recurrence [hazard ratio $(\mathrm{HR})=0.65$ ] and diseaserelated death $(\mathrm{HR}=0.54)$ in patients with locoregional lymph node resection compared to patients who have not undergone locoregional lymph node resection. Neoadjuvant therapy is recommended only if complete resection is not feasible $(6,45)$.

\section{Chemotherapy}

Platinum-based chemotherapy is the gold standard for metastatic ACC, and immunotherapy is the new mode of treatment. In instances of inoperable cases, the primary tumor can be treated with external beam radiation for palliative treatment. Lack of resection with a vena caval thrombus can lead to death. The first report of mitotane describes the destruction of zona glomerulosa and zona reticularis in the adrenal gland of dogs receiving mitotane and thereby demonstrating marked reduction of glucocorticoids and 17 hydroxycorticoids even after ACTH stimulation (46). The First International Randomized trial in locally advanced and Metastatic ACC Treatment (FIRMACT) study established the combination of mitotane with etoposide-doxorubicin-cisplatin as the standard therapy for advanced diseases (47). Another study reported that adjuvant mitotane is associated with decreased recurrence and decreased survival $(4,48,49)$. However, the patients in the mitotane group had the worst prognostic factors such as more stage IV cases and more secreting tumors. Hypercortisolism is treated with mitotane, which is an adrenolytic with inhibitory effect on adrenal steroidogenesis. The usual dosage of mitotane is $500 \mathrm{mg}$. For recurrent ACC, mitotane with cisplatin is considered with 3 cycles of $80 \mathrm{mg} / \mathrm{m}^{2}$ cisplatin (43).

Mitotane can cause steroid abnormalities via CYP11B1 and CYP11A1 inhibition, CYP3A4 and increased 6-betahydroxylation of cortisol, and induction of cortisol binding globulin. Side effects of the use of mitotane include gastrointestinal and neurologic abnormalities. Cortisol supplementation is required for the gastrointestinal adverse effect of mitotane. Contraception is recommended during mitotane therapy. Vitamin K antagonist supplementation and combined antitumor therapies are recommended with mitotane treatment. Mitotane plasma monitoring with the objective of reaching 14 to $20 \mathrm{mg} / \mathrm{dL}$ is recommended for ACC management, as it has a high volume of distribution 
and low clearance due to it being a lipophilic drug with fatty tissue concentration 200 times as high as in plasma concentration. Adaptive dosing of the individual based on age, sex, body mass index, lean body mass, and renal function is recommended (50).

Steroidogenesis enzyme blockers such as ketoconazole and metyrapone are also used in the management of the disease. Glucocorticoid antagonist such as mifepristone is used as a glucosteroid blocker. Hyperaldosteronism is controlled by spironolactone and eplerenone. Sodium and renal function must be monitored during treatment with spironolactone. Androgen excess is treated with spironolactone, bicalutamide, or flutamide, while estrogen excess is treated with estrogen receptor antagonist and aromatase inhibitors (51). Targeted therapy may also be employed for the management of ACC, although studies on targeted therapy and salvage treatment have not shown promising results. The first trials targeted epidermal growth factor receptor gefitinib and a combination of erlotinib and gemcitabine. Among vascular endothelial growth factors, sunitinib exhibited modest anti-tumor effect in a Phase 2 trial. VEGF should be administered in patients without mitotane treatment $(50,51)$.

\section{Radiation therapy}

Radiation therapy is used for palliation and as adjuvant therapy, and it is not a mainstay of treatment, given that cancer cells are not easily killed by radiation. The timing of radiotherapy as an adjuvant is usually 4 to 6 weeks after the initial surgical resection. Loco-guided regional therapy including interventional radiology technique is used to control tumor growth and improve secretory status. Other therapies including radiofrequency ablation, cryotherapy, and transarterial chemoembolization are effective in curing small metastatic lesions in the liver, lung, and bone tissue $(50,51)$.

\section{Surveillance}

Patients should be followed up every 3 months during and after initial treatment. After the first 2 years, the imaging interval is increased. Follow-up is recommended up to 10 years after the surgical resection of the primary tumor. Genetic counseling is recommended, as ACC is associated with a germline mutation in TP53 and Li-Fraumeni syndrome, which includes MLH1, MSH2, MSH6, PMS2 mutations $(50,51)$.

\section{Future perspective}

Personalized therapy, including identifying the actionable target in each patient, is the future of ACC management. The knowledge base of ACC is evolving based on the basic science and clinical trials conducted by worldwide groups such as COMITE of France, ENSAT of Europe, the TCGA project, and the American Australian Asian Adrenal Alliance. The first industry-sponsored randomized placebo-controlled double-blind Phase 3 clinical trial (GALACTICC) also brought about knowledge to advance the management of the trial. Beta-catenin signaling, p53/Rb pathway, chromatin remodeling, targeted gene therapy on CTNNB1, and TP53 are promising avenues for the study. The rarity of ACC makes Phase 3 clinical trials challenging. Future studies should aim at clear molecular and clinical standardization. Recommended therapeutic strategies should be prospectively recorded. Mitotane therapeutic levels should be recorded at the time of new therapeutic evaluations. Phase 1 studies in patients with ACC must be implemented. In Phase 3 studies, implementation of stratification of mitotane receptors as well as prognostic parameters must be considered. The FIRM ACT and GALACCTIC trial are some of the new studies that are aiming to improve the standard of care of ACC.

\section{Acknowledgments}

Funding: None.

\section{Footnote}

Conflicts of Interest: All authors have completed the ICMJE uniform disclosure form (available at http://dx.doi. org/10.21037/tcr.2019.12.28). The authors have no conflicts of interest to declare.

Ethical Statement: The authors are accountable for all aspects of the work in ensuring that questions related to the accuracy or integrity of any part of the work are appropriately investigated and resolved.

Open Access Statement: This is an Open Access article distributed in accordance with the Creative Commons Attribution-NonCommercial-NoDerivs 4.0 International License (CC BY-NC-ND 4.0), which permits the noncommercial replication and distribution of the article with the strict proviso that no changes or edits are made and the 
original work is properly cited (including links to both the formal publication through the relevant DOI and the license). See: https://creativecommons.org/licenses/by-nc-nd/4.0/.

\section{References}

1. Bilimoria KY, Shen WT, Elaraj D, et al. Adrenocortical carcinoma in the United States: treatment utilization and prognostic factors. Cancer 2008;113:3130-6.

2. Else T. Association of adrenocortical carcinoma with familial cancer susceptibility syndromes. Mol Cell Endocrinol 2012;351:66-70.

3. Hsing AW, Nam JM, Co Chien HT, et al. Risk factors for adrenal cancer: an exploratory study. Int J Cancer 1996;65:432-6.

4. Luton JP, Cerdas S, Billaud L, et al. Clinical features of adrenocortical carcinoma, prognostic factors, and the effect of mitotane therapy. N Engl J Med 1990;322:1195-201.

5. Else T, Kim AC, Sabolch A, et al. Adrenocortical carcinoma. Endocr Rev 2014;35:282-326.

6. Puglisi S, Perotti P, Pia A, et al. Adrenocortical Carcinoma with Hypercortisolism. Endocrinol Metab Clin North Am 2018;47:395-407.

7. Pinto EM, Billerbeck AEC, Villares MCBF, et al. Founder effect for the highly prevalent $\mathrm{R} 337 \mathrm{H}$ mutation of tumor suppressor p53 in Brazilian patients with adrenocortical tumors. Arq Bras Endocrinol Metabol 2004;48:647-50.

8. Shifrin A. The History of the Adrenal Gland. Adrenal Tumors Web site. Available online: http://www. adrenaltumors.org/history, accessed October 23, 2019.

9. Cushing H. The Pituitary Body and Its Disorders, Clinical States Produced by Disorders of the Hypophysis Cerebri. An Amplification of the Harvey Lecture for December, 1910. J.B. Lippincott Company, Philadelphia, 1912.

10. Conn JW. Presidential address. I. Painting background. II. Primary aldosteronism, a new clinical syndrome. J Lab Clin Med 1955;45:3-17.

11. Phan AT. Adrenal cortical carcinoma-review of current knowledge and treatment practices. Hematol Oncol Clin North Am 2007;21:489-507; viii.

12. Nakamura Y, Yamazaki Y, Felizola SJ, et al. Adrenocortical carcinoma: review of the pathologic features, production of adrenal steroids, and molecular pathogenesis. Endocrinol Metab Clin North Am 2015;44:399-410.

13. Bertherat J, Bertagna X. Pathogenesis of adrenocortical cancer. Best Pract Res Clin Endocrinol Metab 2009;23:261-71.

14. Bonnet-Serrano F, Bertherat J. Genetics of tumors of the adrenal cortex. Endocr Relat Cancer 2018;25:R131-R152.

15. Sbiera S, Schmull S, Assie G, et al. High diagnostic and prognostic value of steroidogenic factor-1 expression in adrenal tumors. J Clin Endocrinol Metab 2010;95:E161-71.

16. Zheng S, Cherniack AD, Dewal N, et al. Comprehensive pan-genomic characterization of adrenocortical carcinoma. Cancer Cell 2016;29:723-36.

17. Fassnacht M, Kroiss M, Allolio B. Update in adrenocortical carcinoma. J Clin Endocrinol Metab 2013;98:4551-64.

18. Fassnacht M, Allolio B. Clinical management of adrenocortical carcinoma. Best Pract Res Clin Endocrinol Metab 2009;23:273-89.

19. Fassnacht M, Allolio B. Epidemiology of adrenocortical carcinoma. In: Hammer G, Else T. editors. Adrenocortical Carcinoma, 1st ed. New York: Springer, 2010:23-9.

20. Abiven G, Coste J, Groussin L, et al. Clinical and biological features in the prognosis of adrenocortical cancer: poor outcome of cortisol-secreting tumors in a series of 202 consecutive patients. J Clin Endocrinol Metab 2006;91:2650-5.

21. Sturgeon C, Shen WT, Clark OH, et al. Risk assessment in 457 adrenal cortical carcinomas: how much does tumor size predict the likelihood of malignancy? J Am Coll Surg 2006;202:423-30.

22. Bharwani N, Rockall AG, Sahdev A, et al. Adrenocortical carcinoma: the range of appearances on CT and MRI. AJR Am J Roentgenol 2011;196:W706-14.

23. Mansmann G, Lau J, Balk E, et al. The clinically inapparent adrenal mass: update in diagnosis and management. Endocr Rev 2004;25:309-40.

24. Terzolo M, Stigliano A, Chiodini I, et al. AME position statement on adrenal incidentaloma. Eur J Endocrinol 2011;164:851-70.

25. Gröndal S, Eriksson B, Hagenäs L, et al. Steroid profile in urine: a useful tool in the diagnosis and follow up of adrenocortical carcinoma. Acta Endocrinol (Copenh) 1990;122:656-63.

26. Arlt $W$, Biehl M, Taylor AE, et al. Urine steroid metabolomics as a biomarker tool for detecting malignancy in adrenal tumors. J Clin Endocrinol Metab 2011;96:3775-84.

27. Egbert N, Elsayes KM, Azar S, et al. Computed tomography of adrenocortical carcinoma containing macroscopic fat. Cancer Imaging 2010;10:198-200.

28. Groussin L, Bonardel G, Silvéra S, et al. 18F-Fluorodeoxyglucose positron emission tomography for the diagnosis of adrenocortical tumors: a prospective study in 77 operated patients. J Clin Endocrinol Metab 
2009;94:1713-22.

29. Hahner S, Stuermer A, Kreissl M, et al. [123 I] Iodometomidate for molecular imaging of adrenocortical cytochrome P450 family 11B enzymes. J Clin Endocrinol Metab 2008;93:2358-65.

30. Assié G, Jouinot A, Fassnacht M, et al. Value of molecular classification for prognostic assessment of adrenocortical carcinoma. JAMA Oncol 2019. DOI: 10.1001/ jamaoncol.2019.1558.

31. Libé R, Borget I, Ronchi CL, et al. Prognostic factors in Stage III-IV adrenocortical carcinomas (ACC): an European Network for the Study of Adrenal Tumor (ENSAT) study. Ann Oncol 2015;26:2119-25.

32. Beuschlein F, Weigel J, Saeger W, et al. Major prognostic role of Ki67 in localized adrenocortical carcinoma after complete resection. J Clin Endocrinol Metab 2015;100:841-9.

33. Bowlby LS, DeBault LE, Abraham SR. Flow cytometric analysis of adrenal cortical tumor DNA. Relationship between cellular DNA and histopathologic classification. Cancer 1986;58:1499-505.

34. Amberson JB, Vaughan ED, Gray GF, et al. Flow cytometric determination of nuclear DNA content in benign adrenal pheochromocytomas. Urology 1987;30:102-4.

35. Stephan EA, Chung T-H, Grant CS, et al. Adrenocortical carcinoma survival rates correlated to genomic copy number variants. Mol Cancer Ther 2008;7:425-31.

36. Barreau O, Assié G, Wilmot-Roussel H, et al. Identification of a $\mathrm{CpG}$ island methylator phenotype in adrenocortical carcinomas. J Clin Endocrinol Metab 2013;98:E174-84.

37. de Reyniès A, Assié G, Rickman DS, et al. Gene expression profiling reveals a new classification of adrenocortical tumors and identifies molecular predictors of malignancy and survival. J Clin Oncol 2009;27:1108-15.

38. Fragoso MCBV, Almeida MQ, Mazzuco TL, et al. Combined expression of BUB1B, DLGAP5, and PINK1 as predictors of poor outcome in adrenocortical tumors: validation in a Brazilian cohort of adult and pediatric patients. Eur J Endocrinol 2012;166:61-7.

39. Tömböl Z, Szabó PM, Molnár V, et al. Integrative

Cite this article as: Thampi A, Shah E, Elshimy G, Correa R. Adrenocortical carcinoma: a literature review. Transl Cancer Res 2020;9(2):1253-1264. doi: 10.21037/tcr.2019.12.28 molecular bioinformatics study of human adrenocortical tumors: microRNA, tissue-specific target prediction, and pathway analysis. Endocr Relat Cancer 2009;16:895-906.

40. Doghman M, El Wakil A, Cardinaud B, et al. Regulation of insulin-like growth factor-mammalian target of rapamycin signaling by microRNA in childhood adrenocortical tumors. Cancer Res 2010;70:4666-75.

41. Libé R. Adrenocortical carcinoma (ACC): diagnosis, prognosis, and treatment. Front Cell Dev Biol 2015;3:45.

42. Baudin E, Endocrine Tumor Board of Gustave Roussy. Adrenocortical carcinoma. Endocrinol Metab Clin North Am 2015;44:411-34.

43. Postlewait LM, Ethun CG, Tran TB, et al. Outcomes of adjuvant mitotane after resection of adrenocortical carcinoma: A 13-institution study by the US Adrenocortical Carcinoma Group. J Am Coll Surg 2016;222:480-90.

44. Tella SH, Kommalapati A, Yaturu S, et al. Predictors of survival in adrenocortical carcinoma: an analysis from the National Cancer Database. J Clin Endocrinol Metab 2018;103:3566-73.

45. Kendrick ML, Lloyd R, Erickson L, et al. Adrenocortical carcinoma: surgical progress or status quo? Arch Surg 2001;136:543-9.

46. Nelson AA, Woodard G. Severe adrenal cortical atrophy (cytotoxic) and hepatic damage produced in dogs by feeding 2,2-bis(parachlorophenyl)-1,1-dichloroethane (DDD or TDE). Arch Pathol (Chic) 1949;48:387-94.

47. Fassnacht M, Terzolo M, Allolio B, et al. Combination chemotherapy in advanced adrenocortical carcinoma. $\mathrm{N}$ Engl J Med 2012;366:2189-97.

48. Terzolo M, Angeli A, Fassnacht M, et al. Adjuvant mitotane treatment for adrenocortical carcinoma. N Engl J Med 2007;356:2372-80.

49. Haak HR, van Seters AP, Moolenaar AJ. Mitotane therapy of adrenocortical carcinoma. N Engl J Med 1990;323:758.

50. Miller BS, Else T, AACE Adrenal Scientific Committee. Personalized care of patients with adrenocortical carcinoma: a comprehensive approach. Endocr Pract 2017;23:705-15.

51. Jasim S, Habra MA. Management of adrenocortical carcinoma. Curr Oncol Rep 2019;21:20. 